\title{
Semi-stability of embedded solitons in the general fifth-order $\mathrm{KdV}$ equation
}

\author{
Yu Tan ${ }^{\mathrm{a}}$, Jianke Yang ${ }^{\mathrm{a}, *}$, Dmitry E. Pelinovsky ${ }^{\mathrm{b}}$ \\ a Department of Mathematics and Statistics, University of Vermont, Burlington, VT 05401, USA \\ ${ }^{\mathrm{b}}$ Department of Mathematics, McMaster University, Hamilton, Ont., Canada L8S 4K1
}

Received 26 September 2001; received in revised form 10 January 2002; accepted 6 February 2002

\begin{abstract}
Evolution of perturbed embedded solitons in the general Hamiltonian fifth-order Korteweg-de Vries (KdV) equation is studied. When an embedded soliton is perturbed, it sheds a one-directional continuous-wave radiation. It is shown that the radiation amplitude is not minimal in general. A dynamical equation for velocity of the perturbed embedded soliton is derived. This equation shows that a neutrally stable embedded soliton is in fact semi-stable. When the perturbation increases the momentum of the embedded soliton, the perturbed state approaches asymptotically the embedded soliton, while when the perturbation reduces the momentum of the embedded soliton, the perturbed state decays into radiation. Classes of initial conditions to induce soliton decay or persistence are also determined. Our analytical results are confirmed by direct numerical simulations of the fifth-order KdV equation.

() 2002 Elsevier Science B.V. All rights reserved.
\end{abstract}

\section{Introduction}

Embedded solitons are solitary wave solutions of nonlinear evolution equations which reside at discrete points inside the continuous spectrum of the linear wave system. The existence of such waves has been known in various physical systems such as the fifth-order Korteweg-de Vries (KdV) equations [1-3], extended nonlinear Schrödinger equations [4,5], coupled KdV equations [6], second-harmonic-generation (SHG) system [7], massive Thirring model [8,9], three-wave system [10], and many others [11,12]. In [7], such waves were given the name "embedded solitons", and their distinct semi-stability property was revealed on heuristic ground. This semi-stability means that when a perturbation increases a certain positive-definite quantity (energy or momentum) associated with the embedded soliton, then the perturbed state approaches asymptotically the embedded soliton. However, when the perturbation decreases energy (momentum) of the embedded soliton, the perturbed state decays into radiation.

The semi-stability property was later proved rigorously for embedded solitons in the perturbed integrable fifth-order KdV equation [13] and in the generalized SHG system [14]. The method in [13] follows the soliton perturbation technique and describes embedded solitons as critical points of a first-order dynamical system. Both location and stability of critical points can be studied within the reduced system. The other paper [14] develops the normal form analysis which relies on the known existence and linearized stability properties of embedded solitons. It proves the nonlinear semi-stability of embedded solitons through wave resonance mechanisms. The latter method

\footnotetext{
* Corresponding author. Tel.: +1-802-656-4314; fax: +1-802-656-2552.
}

E-mail address: jyang@emba.uvm.edu (J. Yang). 
does not rely on the integrability of the original system, and it can be extended to any embedded-soliton-bearing system under certain assumptions.

Nonlinear semi-stability of embedded solitons is an interesting phenomenon because it occurs beyond the linear stability. The linear stability of solitary waves in the fifth-order KdV equation was studied by using the energy-momentum methods [15,16] and the symplectic Evans matrix methods [17]. A solitary wave is linearly unstable if the linearization operator possesses an eigenvalue with a positive real part. If all eigenvalues lie on the imaginary axis, the wave is called neutrally stable. A neutrally stable wave can, however, still be unstable due to algebraic instabilities [18]. For embedded solitons, the situation is different. Single-hump embedded solitons are generally neutrally stable in the linearized problem. However, a discrete zero eigenvalue of the linearization operator is embedded inside the continuous spectrum of this operator. Because of this, a nonlinear resonance between the embedded zero eigenvalue and the continuous spectrum can tunnel energy of a perturbed embedded soliton into continuous-wave radiation. The energy loss does not always destroy the embedded soliton though. If the perturbation increases the energy (momentum) of the embedded soliton, then the radiation becomes weaker and weaker as the perturbed state asymptotically approaches the embedded soliton. But if the perturbation decreases the energy (momentum) of the embedded soliton, the radiation becomes stronger and stronger, and the embedded soliton is then destroyed. Thus, the semi-stability of embedded solitons is an intrinsically nonlinear phenomenon beyond linear stability.

Besides single-humped embedded soliton in the fifth-order KdV equation, multi-humped embedded solitons may also exist [3,19]. However, they are typically linearly unstable [11,12]. The nonlinear semi-stability may occur in the system only if the linear instability is suppressed. Thus, it makes sense to consider here only neutrally stable single-humped embedded solitons.

We emphasize that semi-stability is not the same as instability. By controlling the energy (momentum) of the initial perturbation, we can induce either asymptotic persistence of the embedded soliton or its rapid disappearance. This is an ideal mechanism for switching (quantization) applications.

In this paper, we study the evolution of perturbed neutrally stable embedded solitons in a general Hamiltonian fifth-order KdV equation. We use the normal form analysis developed in [14] but simplify many statements and proofs. We show that when an embedded soliton in the fifth-order KdV equation is perturbed, it sheds the continuous-wave radiation in front of the soliton. The radiation amplitude is not minimal in general. We also derive the velocity equation for the perturbed state which proves the semi-stability property of embedded solitons. In addition, we determine what initial condition leads to soliton decay, and what initial condition leads to soliton persistence. Numerical simulations of the fifth-order KdV equation show excellent agreement with the analytical predictions.

\section{Tail amplitudes of symmetric nonlocal waves}

We consider the general Hamiltonian fifth-order KdV equation

$$
u_{t}+u_{x x x}+u_{x x x x x}+[N(u)]_{x}=0
$$

where the nonlinear term $N(u)$ is of the form

$$
N(u)=\alpha_{0} u^{2}+\alpha_{1} u u_{x x}+\alpha_{2} u_{x}^{2}+\alpha_{3} u^{3} .
$$

The equations above are Hamiltonian if and only if $\alpha_{1}=2 \alpha_{2}$ [13]. The conserved Hamiltonian functional $H(u)$ is

$$
H(u)=\int_{-\infty}^{\infty}\left[\frac{\alpha_{0}}{3} u^{3}-\frac{1}{2} u_{x}^{2}+\frac{1}{2} u_{x x}^{2}-\frac{\alpha_{1}}{2} u u_{x}^{2}+\frac{\alpha_{3}}{4} u^{4}\right] \mathrm{d} x .
$$

When Eq. (2.1) is Hamiltonian, it also conserves a positive-definite quantity

$$
P(u)=\int_{-\infty}^{\infty} u^{2} \mathrm{~d} x,
$$


which is interpreted as momentum. Whether Eq. (2.1) is Hamiltonian or not, it always conserves the quantity $M(u)=\int_{-\infty}^{\infty} u \mathrm{~d} x$, which is interpreted as mass.

We look for moving stationary solutions in Eq. (2.1) of the form

$$
u(x, t)=U(x-c t) \equiv U(\xi),
$$

where $c$ is the wave velocity. Substituting Eq. (2.5) into (2.1) and integrating once, we obtain the ordinary differential equation (ODE) for $U(\xi)$ as

$$
U_{\xi \xi \xi \xi}+U_{\xi \xi}-c U+N(U)=0
$$

subject to zero boundary conditions at infinity: $\lim _{|\xi| \rightarrow \infty} U(\xi)=0$. When $U \rightarrow 0$, Eq. (2.6) becomes linear, and its characteristic equation is quartic. The roots of this characteristic equation are $\pm \mathrm{i} k$ and $\pm \kappa$, where

$$
k=k(c)=\sqrt{\frac{1}{2}(\sqrt{1+4 c}+1)}, \quad \kappa=\kappa(c)=\sqrt{\frac{1}{2}(\sqrt{1+4 c}-1)} .
$$

When $c>0$, roots $\pm \mathrm{i} k$ are imaginary and $\pm \kappa$ real; when $0>c>-1 / 4$, all four roots are imaginary; when $c<-1 / 4$, all four roots are complex. The embedded soliton, if it exists, arises from a saddle-node bifurcation [19], when the stable-unstable manifolds (real roots) correspond to the exponential tails of the embedded soliton, while the center manifold (imaginary roots) correspond to the tails of the continuous-wave radiation whose amplitude vanishes. Therefore, the embedded soliton may exist in Eq. (2.6) only for $c>0$. We notice that nonembedded solitary waves with oscillatory and decaying tails may exist in Eq. (2.6) for $c<-1 / 4$ [20], but such solutions are beyond the scope of this paper.

Only symmetric embedded solitons of Eq. (2.6) are considered here. Based on energy flux consideration, it is generally believed that asymmetric embedded solitons of Eq. (2.6) do not exist [21,22]. If the system (2.1) is the perturbed integrable fifth-order KdV equation, the nonexistence of asymmetric embedded solitons in Eq. (2.6) was proved in [13].

When $c>0$, the tail amplitude of symmetric nonlocal waves of Eq. (2.6) is given asymptotically as

$$
U(\xi ; c, \delta) \rightarrow r(c, \delta) \sin (k|\xi|+\delta), \quad|\xi| \rightarrow \infty .
$$

Here $r$ is the amplitude of the oscillatory tail, and $\delta$ the tail phase. Embedded solitons can be found numerically by a shooting method for Eq. (2.6) with $U^{\prime}(0)=U^{\prime \prime \prime}(0)=0$. Parameters of the shooting method $U(0)$ and $U^{\prime \prime}(0)$ satisfy only one condition that removes the exponentially growing term $\sim \mathrm{e}^{\kappa|\xi|}$. It implies that one parameter in symmetric nonlocal wave solutions of Eq. (2.6) is free in addition to the wave velocity $c$. We choose this free parameter to be the tail phase $\delta$. The tail amplitude $r(c, \delta)$ may vanish at certain discrete velocity values $c=c_{\mathrm{ES}}$ (in such cases, the phase $\delta$ becomes irrelevant). When it happens, we get embedded solitons. The $\operatorname{sech}^{2}$ embedded solitons in Eq. (2.1) has been investigated comprehensively in [1,13], and a family of one, two, and $\infty$ embedded solitons have been shown to exist in different parameter regions. Single-humped and multi-humped solutions of (2.6) were studied also in [19]. We assume here the generic case of a co-dimension one bifurcation, when the embedded-soliton velocity $c_{\mathrm{ES}}$ is a simple zero of the tail amplitude $r(c, \delta)$, i.e.,

$$
r(c, \delta)=R(\delta)\left(c-c_{\mathrm{ES}}\right)+\mathrm{O}\left(c-c_{\mathrm{ES}}\right)^{2},
$$

where

$$
R(\delta)=\frac{\partial r}{\partial c}\left(c_{\mathrm{ES}}, \delta\right) \neq 0
$$

is the slope of the tail amplitude $r$ of the symmetric nonlocal waves at the embedded-soliton velocity $c_{\mathrm{ES}}$ and phase $\delta$.

In the rest of this section, we derive the analytical expression for the tail amplitude $r(c, \delta)$ when the nonlocal wave velocity $c$ is close to the embedded-soliton velocity $c_{\mathrm{ES}}$. For this purpose, we expand the nonlocal solution $U(\xi ; c, \delta)$ as a perturbation series:

$$
U(\xi ; c, \delta)=U_{\mathrm{ES}}(\xi)+\left(c-c_{\mathrm{ES}}\right) U_{1}(\xi ; \delta)+\mathrm{O}\left(c-c_{\mathrm{ES}}\right)^{2} .
$$


When this expansion is substituted into Eq. (2.6), the function $U_{1}(\xi ; \delta)$ is found to satisfy the following inhomogeneous linear equation:

$$
\mathcal{L} U_{1}(\xi ; \delta)=U_{\mathrm{ES}}(\xi)
$$

where $\mathcal{L}$ is the linearization operator of Eq. (2.6) at embedded-soliton velocity $c_{\mathrm{ES}}$, i.e.,

$$
\mathcal{L}=\frac{\mathrm{d}^{4}}{\mathrm{~d} \xi^{4}}+\frac{\mathrm{d}^{2}}{\mathrm{~d} \xi^{2}}-c_{\mathrm{ES}}+2 \alpha_{0} U_{\mathrm{ES}}+3 \alpha_{3} U_{\mathrm{ES}}^{2}+\alpha_{1} \frac{\mathrm{d}^{2} U_{\mathrm{ES}}}{\mathrm{d} \xi^{2}}+\alpha_{1} \frac{\mathrm{d}}{\mathrm{d} \xi}\left(U_{\mathrm{ES}} \frac{\mathrm{d}}{\mathrm{d} \xi}\right) .
$$

Here the Hamiltonian condition $\alpha_{1}=2 \alpha_{2}$ has been utilized. Note that the operator $\mathcal{L}$ is self-adjoint in the Hamiltonian case.

In order to solve the inhomogeneous equation (2.12), we need to know homogeneous solutions. Operator $\mathcal{L}$ has four homogeneous solutions $\psi_{n}(1 \leq n \leq 4)$. The first solution is symmetric and bounded with asymptotic behavior

$$
\psi_{1}(\xi) \rightarrow \sin \left(k_{r}|\xi|+\delta_{\mathrm{s}}\right), \quad|\xi| \rightarrow \infty
$$

where $k_{r} \equiv k\left(c_{\mathrm{ES}}\right)$ is the resonant wavenumber and $\delta_{\mathrm{s}}$ is the tail phase. The second solution is anti-symmetric and bounded with asymptotic behavior

$$
\psi_{2}(\xi) \rightarrow \sin \left(k_{r} \xi \pm \delta_{\mathrm{a}}\right), \quad \xi \rightarrow \pm \infty
$$

where $\delta_{\mathrm{a}}$ is the tail phase. The third solution is anti-symmetric and localized, $\psi_{3}(\xi)=U_{\mathrm{ES}}^{\prime}(\xi)$. It is related to spatial translational invariance of the system (2.1). The last solution $\psi_{4}(\xi)$ is symmetric and unbounded.

Now we can solve the inhomogeneous equation (2.12) for $U_{1}$. Since we only consider symmetric nonlocal waves, the correction term $U_{1}(\xi)$ is symmetric as well. Note that $\partial U\left(\xi ; c_{\mathrm{ES}}, \delta\right) / \partial c$ is an inhomogeneous solution of Eq. (2.12) for any $\delta$ values. This can be seen by differentiating Eq. (2.6) with respect to $c$, and then setting $c$ as $c_{\mathrm{ES}}$. Thus the general bounded symmetric solution of Eq. (2.12) can be written as

$$
U_{1}(\xi)=\frac{\partial U}{\partial c}\left(\xi ; c_{\mathrm{ES}}, \delta_{0}\right)+\gamma_{1} \psi_{1}(\xi)
$$

Here $\delta_{0}$ is any fixed phase, and $\gamma_{1}$ is an arbitrary constant. The homogeneous solutions $\psi_{2}(\xi)$ and $\psi_{3}(\xi)$ are not included as they are anti-symmetric, while the solution $\psi_{4}(\xi)$ is unbounded.

The asymptotic oscillatory behavior of a general $U_{1}$ solution (2.16) can be obtained from Eqs. (2.8) and (2.14) as

$$
U_{1}(\xi ; \delta) \rightarrow R\left(\delta_{0}\right) \sin \left(k_{r}|\xi|+\delta_{0}\right)+\gamma_{1} \sin \left(k_{r}|\xi|+\delta_{\mathrm{s}}\right), \quad|\xi| \rightarrow \infty
$$

where function $R(\delta)$ is defined by Eq. (2.10). On the other hand, expanding the tail asymptotics (2.8) of the nonlocal wave $U(\xi ; c, \delta)$ into a power series of $c-c_{\mathrm{ES}}$ and then comparing it with Eq. (2.11), we conclude that the asymptotic behavior of a general $U_{1}$ solution should also be

$$
U_{1}(\xi) \rightarrow R(\delta) \sin \left(k_{r}|\xi|+\delta\right), \quad|\xi| \rightarrow \infty .
$$

For convenience, we fix here $\delta_{0}$ as $\delta_{0}=\delta_{\mathrm{s}}+\pi / 2$ and then equate the two asymptotics (2.17) and (2.18). As a result, we find that

$$
R(\delta)=\frac{R\left(\delta_{\mathrm{s}}+\pi / 2\right)}{\sin \left(\delta-\delta_{\mathrm{s}}\right)},
$$

and $\gamma_{1}=R\left(\delta_{\mathrm{s}}+\pi / 2\right) \cot \left(\delta-\delta_{\mathrm{s}}\right)$. Consequently, to leading order in $c-c_{\mathrm{ES}}$, the tail amplitude $r$ of symmetric nonlocal waves is

$$
r(c, \delta)=\frac{R\left(\delta_{\mathrm{S}}+\pi / 2\right)}{\sin \left(\delta-\delta_{\mathrm{S}}\right)}\left(c-c_{\mathrm{ES}}\right)+\mathrm{O}\left(c-c_{\mathrm{ES}}\right)^{2} .
$$


From this formula, we conclude that at a given velocity $c$ close to $c_{\mathrm{ES}}$, the tail amplitude $|r|$ of symmetric nonlocal waves is minimal at the value $\delta=\delta_{\min }$, where

$$
\delta_{\min }=\left(\delta_{\mathrm{s}}+\frac{\pi}{2}\right) \bmod (\pi) .
$$

Formulae (2.20) and (2.21) are the main results of this section.

\section{Dynamics of embedded solitons under perturbations}

In this section, we study dynamics of a linearly neutrally stable embedded soliton under small perturbations. We assume that the linearization operator has no unstable eigenvalues. This assumption is necessary as the weak nonlinear semi-stability would be ineffective in the presence of strong linear instability. We also assume that the linearization operator has no discrete nonzero embedded eigenvalues. This assumption is necessary as the nonzero embedded eigenmodes may be in resonance with the continuous spectrum through nonlinear coupling, thus affecting the dynamics of embedded solitons. Thirdly, we assume that the zero eigenvalue corresponds to the localized eigenfunction $U_{\mathrm{ES}}^{\prime}(\xi)$ and has algebraic multiplicity 2 with an associated eigenfunction $\partial U / \partial c(\xi ; \delta)$. For embedded solitons, the zero eigenvalue is always embedded into the continuous spectrum of the linearization operator. Eigenvalues that correspond to localized eigenfunctions occur as zeros of the Evans function (a determinant of scattering coefficients) [17]. The algebraic multiplicity of eigenvalues is defined as the multiplicity of zeros of the Evans function. Thus, our last assumption is that the Evans function has a double zero at $\lambda=0$ of the linearized operator.

We will use below the internal perturbation analysis described in [14] (see also [18]). The idea is to recognize that under small perturbations, the eigenfunctions $U_{\mathrm{ES}}^{\prime}(\xi)$ and $\partial U / \partial c(\xi ; \delta)$ for the double embedded eigenvalue $\lambda=0$ of the linearized problem renormalize the location and velocity of the embedded soliton. For small perturbations, the velocity $c(t)$ of the embedded soliton changes on a slow time scale. We will derive a dynamical equation for $c(t)$ by separating the slow and fast changes in evolution of a perturbed embedded soliton.

In the moving coordinate,

$$
\xi=x-\int_{0}^{t} c \mathrm{~d} t-x_{0},
$$

the fifth-order KdV equation (2.1) can be written as

$$
u_{t}-c u_{\xi}+u_{\xi \xi \xi}+u_{\xi \xi \xi \xi \xi}+[N(u)]_{\xi}=0 .
$$

We expand the perturbed embedded soliton and its slowly varying velocity into the following perturbation series:

$$
u(\xi, t)=U_{\mathrm{ES}}(\xi)+\epsilon c_{1}(T) u_{1}(\xi, t)+\epsilon^{2} u_{2}(\xi, t, T)+\mathrm{O}\left(\epsilon^{3}\right),
$$

and

$$
c(T)=c_{\mathrm{ES}}+\epsilon c_{1}(T)+\mathrm{O}\left(\epsilon^{2}\right),
$$

where $T=\epsilon t$, and $\epsilon$ is a small parameter. At order $\epsilon$, we obtain the governing equation for $u_{1}(\xi, t)$ as

$$
u_{1 t}+\left[\mathcal{L} u_{1}\right]_{\xi}=U_{\mathrm{ES}}^{\prime}(\xi)
$$

where $\mathcal{L}$ is the same linearization operator as defined in Eq. (2.13). The initial condition for Eq. (3.5) can be obtained from Eq. (3.3) as

$$
u_{1}(\xi, 0)=\frac{u(\xi, 0)-U_{\mathrm{ES}}(\xi)}{\epsilon c_{1}(0)}
$$


The initial value for the soliton velocity $c_{1}(0)$ can be found by projecting the initial deviation $u(\xi, 0)-U_{\mathrm{ES}}(\xi)$ onto $\partial U\left(\xi ; c_{\mathrm{ES}}, \delta_{\mathrm{a}}\right) / \partial c$, where the phase $\delta_{\mathrm{a}}$ is given by (2.15). The projection is based on the spectral decomposition developed in Appendix A (see (A.4)) and is given by

$$
\epsilon c_{1}(0)=\frac{\int_{-\infty}^{\infty} U_{\mathrm{ES}}(\xi)\left[u(\xi, 0)-U_{\mathrm{ES}}(\xi)\right] \mathrm{d} \xi}{\int_{-\infty}^{\infty} U_{\mathrm{ES}}(\xi)(\partial U / \partial c)\left(\xi ; c_{\mathrm{ES}}, \delta_{\mathrm{a}}\right) \mathrm{d} \xi} .
$$

Next, we solve the inhomogeneous equation (3.5). We adopt a less formal but more intuitive approach here. A more rigorous calculation of the same results is presented in Appendix A.

The inhomogeneous term in Eq. (3.5) acts as a driving localized force. The homogeneous part at large $|\xi|$ values supports oscillatory solutions with wavenumber $k_{r}$. Thus, due to forcing and resonance, these oscillatory tails will be excited over time. The group velocity of these oscillatory tails in the moving frame (3.1) can be found from the dispersion relation as $c_{\mathrm{gr}}=2 k_{r}^{2}\left(2 k_{r}^{2}-1\right)$, which is always positive since $c_{\mathrm{ES}}>0$ and $k_{r}>1$. Thus, these oscillatory tails always appear ahead of the embedded soliton. Behind the embedded soliton, there is the possibility that a flat shelf may develop as in the perturbed KdV equation [23,24] (see also [18]). If a shelf develops, it moves to the region $x \ll-1$ at the velocity $-c_{\mathrm{ES}}$ in the moving coordinate system (3.1).

Thus, at large times $t \gg 1$, the boundary conditions for the solution $u_{1}(\xi, t)$ are

$$
u_{1}(\xi, t) \rightarrow \begin{cases}R_{\mathrm{rad}} \sin \left(k_{r} \xi+\delta_{\mathrm{rad}}\right) H\left(c_{\mathrm{gr}} t-\xi\right), & \xi \gg 1, \\ R_{0} H\left(-\xi-c_{\mathrm{ES}} t\right), & \xi \ll-1,\end{cases}
$$

where $R_{\text {rad }}$ is the oscillatory tail amplitude, $\delta_{\text {rad }}$ is its phase, $R_{0}$ the height of the trailing shelf, and $H(x)$ the step function, i.e., $H=1$ when $x \geq 0$, and $H=0$ otherwise. Below, we determine the tail amplitude $R_{\text {rad }}$ and the phase $\delta_{\text {rad }}$. We also show that the shelf is not excited in the present situation, i.e., $R_{0}=0$.

Our calculations of $R_{0}, R_{\text {rad }}$ and $\delta_{\text {rad }}$ are based on the observation that, as $t$ goes to infinity, the transient part of the solution $u_{1}(\xi, t)$ for Eq. (3.5) dies out, and $u_{1}(\xi, t)$ approaches a steady state $u_{1 \mathrm{~s}}(\xi)$, where

$$
u_{1 \mathrm{~s}}(\xi) \rightarrow \begin{cases}R_{\mathrm{rad}} \sin \left(k_{r} \xi+\delta_{\mathrm{rad}}\right), & \xi \rightarrow \infty \\ R_{0}, & \xi \rightarrow-\infty\end{cases}
$$

This steady-state solution satisfies the same equation (3.5) except that the time derivative in (3.5) is dropped, i.e.,

$$
\left[\mathcal{L} u_{1 \mathrm{~s}}\right]_{\xi}=U_{\mathrm{ES}}^{\prime}(\xi) .
$$

Integration of this equation with respect to $\xi$ gives

$$
\mathcal{L} u_{1 \mathrm{~s}}=U_{\mathrm{ES}}+\eta,
$$

where $\eta$ is a constant. To determine $\eta$, we substitute the boundary condition (3.9) of solution $u_{1 \mathrm{~s}}(\xi)$ at $\xi \gg 1$ into Eq. (3.11) and find that $\eta=0$. Then substitution of the boundary condition (3.9) at $\xi \ll-1$ into Eq. (3.11) readily shows that $R_{0}=0$. Thus, the flat shelf is not excited in the present situation.

Since $R_{0}=\eta=0$, the inhomogeneous equation (3.11) for $u_{1 \mathrm{~s}}(\xi)$ becomes the same as Eq. (2.12). The general bounded solution for $u_{1 \mathrm{~s}}(\xi)$ is

$$
u_{1 \mathrm{~s}}(\xi)=\frac{\partial U}{\partial c}\left(\xi ; c_{\mathrm{ES}}, \delta_{0}\right)+\Gamma_{1} \psi_{1}(\xi)+\Gamma_{2} \psi_{2}(\xi),
$$

where $\delta_{0}$ is any fixed phase and $\Gamma_{1,2}$ are constants. The homogeneous solution $\psi_{3}(\xi)$ is excluded by a simple position normalization of the embedded soliton. The boundary condition of solution (3.12) at infinity can be obtained from Eqs. (2.8), (2.14) and (2.15) as

$$
u_{1 \mathrm{~s}}(\xi) \rightarrow \begin{cases}R\left(\delta_{0}\right) \sin \left(k_{r} \xi+\delta_{0}\right)+\Gamma_{1} \sin \left(k_{r} \xi+\delta_{\mathrm{s}}\right)+\Gamma_{2} \sin \left(k_{r} \xi+\delta_{\mathrm{a}}\right), & \xi \rightarrow \infty \\ -R\left(\delta_{0}\right) \sin \left(k_{r} \xi-\delta_{0}\right)-\Gamma_{1} \sin \left(k_{r} \xi-\delta_{\mathrm{s}}\right)+\Gamma_{2} \sin \left(k_{r} \xi-\delta_{\mathrm{a}}\right), & \xi \rightarrow-\infty\end{cases}
$$


This boundary condition should match condition (3.9) with $R_{0}=0$. For convenience, we fix $\delta_{0}=\delta_{\mathrm{a}}$. Then the matching condition gives the radiation amplitude $R_{\text {rad }}$ and phase $\delta_{\text {rad }}$ as

$$
R_{\text {rad }}=2 R\left(\delta_{\mathrm{a}}\right), \quad \delta_{\mathrm{rad}}=\delta_{\mathrm{a}},
$$

and

$$
\Gamma_{1}=0, \quad \Gamma_{2}=R\left(\delta_{\mathrm{a}}\right) .
$$

Formulae (3.14) are important results of this section. They show that the radiation phase $\delta_{\text {rad }}$ is equal to the phase $\delta_{\mathrm{a}}$ of the anti-symmetric homogeneous solution $\psi_{2}(\xi)$ (see Eq. (2.15)), while the radiation amplitude $R_{\text {rad }}=2 R\left(\delta_{\mathrm{a}}\right)$. Since the minimal tail amplitude of symmetric nonlocal waves occurs at phase $\delta_{\min }=\left(\delta_{\mathrm{s}}+\pi / 2\right) \bmod (\pi)$ (see Eq. (2.21)), and $\delta_{\mathrm{a}} \neq\left(\delta_{\mathrm{s}}+\pi / 2\right) \bmod (\pi)$ in general (see Section 4 for an example), we conclude that the radiation amplitude $R_{\text {rad }}$ generally is not minimal, i.e. $R_{\text {rad }} \not \equiv 2 R\left(\delta_{\min }\right)$. In the numerical work for the KdV equation plus the fifth-order derivative [25, Section 16.6], it was mentioned without proof that radiation tail amplitude was minimal. That statement does not agree with our general analysis. But if the fifth-order KdV equation is integrable, then the relation $\delta_{\mathrm{a}}=\delta_{\mathrm{s}}+\pi / 2$ holds [13], i.e. the radiation amplitude is indeed minimal to the leading order of the perturbation theory for nearly integrable fifth-order $\mathrm{KdV}$ equations.

When the first-order solutions (3.12) and (3.15) are substituted into the perturbation expansion (3.3), the solution can be re-written as

$$
u(\xi, t)=\left\{U\left(\xi ; c, \delta_{\mathrm{a}}\right)+\left(c-c_{\mathrm{ES}}\right) R\left(\delta_{\mathrm{a}}\right) \psi_{2}(\xi)+\mathrm{O}\left[\left(c-c_{\mathrm{ES}}\right)^{2}\right]\right\} H\left(c_{\mathrm{gr}} t-\xi\right), \quad t \gg 1,
$$

where $c(T)$ is given by Eq. (3.4). This solution describes the slow evolution of the perturbed embedded soliton in the fifth-order $\mathrm{KdV}$ equation (2.1), while the fast radiation part produced by a general initial condition for $u(\xi, 0)$ is neglected in the asymptotic limit $t \gg 1$. Solution (3.16) up to order $\mathrm{O}\left(c-c_{\mathrm{ES}}\right)$ consists of a symmetric nonlocal wave $U\left(\xi ; c, \delta_{\mathrm{a}}\right)$ and an anti-symmetric term $\psi_{2}(\xi)$. This anti-symmetric term is generated in the initial-value evolution problem due to the (radiation) boundary condition (3.8) with $R_{0}=0, R_{\mathrm{rad}}=2 R\left(\delta_{\mathrm{a}}\right.$ ), and $\delta_{\mathrm{rad}}=\delta_{\mathrm{a}}$. Since function $\psi_{2}(\xi)$ is anti-symmetric, the radiation amplitude is canceled behind the embedded soliton and is doubled ahead of the soliton. It is also noted that $\psi_{2}(0)=0$, thus the amplitude of solution (3.16) at soliton center $\xi=0$ is then the same as that of the symmetric nonlocal wave $U\left(\xi ; c, \delta_{\mathrm{a}}\right)$. This fact will be used in Section 4 in our comparison between the analytical and numerical results on the amplitudes of perturbed embedded solitons.

When the radiation amplitude $R_{\text {rad }}$ and phase $\delta_{\text {rad }}$ are found, we are ready to derive the dynamical equation for the velocity $c(T)$ of a perturbed embedded soliton. This equation can be derived in several different ways (see [13]). The simplest way is to use the local or global momentum conservation law when the system (2.1) is Hamiltonian. The derivation using the global momentum conservation law (2.4) is presented below. The derivation using the local momentum conservation is contained in Appendix B.

To derive the velocity equation, we substitute the perturbation expansion (3.3) into the momentum integral (2.4). When terms up to order $\epsilon^{2}$ are retained, we get

$$
\frac{\mathrm{d}}{\mathrm{d} t} \int_{-\infty}^{\infty}\left\{U_{\mathrm{ES}}^{2}+2 \epsilon U_{\mathrm{ES}}\left(c_{1} u_{1}+\epsilon u_{2}\right)+\epsilon^{2} c_{1}^{2} u_{1}^{2}\right\} \mathrm{d} \xi=0 .
$$

Keep in mind that solutions $u_{1}(\xi, t)$ and $u_{2}(\xi, t)$ at the center region $\xi \sim \mathrm{O}(1)$ become stationary as $t \gg 1$. As a result, the term involving $u_{2}$ in Eq. (3.17) can be dropped because the integral of the product $U_{\mathrm{ES}}(\xi) u_{2}(\xi, t)$ becomes constant at large times. The stationary solution $u_{1 \mathrm{~s}}(\xi)$ is given by Eqs. (3.12) and (3.15). Thus,

$$
E \equiv \int_{-\infty}^{\infty} U_{\mathrm{ES}} u_{1 \mathrm{~s}} \mathrm{~d} \xi=\int_{-\infty}^{\infty} U_{\mathrm{ES}}(\xi) \frac{\partial U}{\partial c}\left(\xi ; c_{\mathrm{ES}}, \delta_{\mathrm{a}}\right) \mathrm{d} \xi
$$

Lastly, the solution for $u_{1}(\xi, t)$ develops an oscillatory tail ahead of the embedded soliton. This tail has amplitude $R_{\text {rad }}$ given by Eq. (3.14), and it moves at its group velocity $c_{\mathrm{gr}}$. The average energy $\left(u_{1}^{2}\right)$ of the sinusoidal tail is 
$(1 / 2) R_{\text {rad }}^{2}$, i.e., $2 R^{2}\left(\delta_{\mathrm{a}}\right)$. Thus,

$$
\Gamma \equiv \frac{\mathrm{d}}{\mathrm{d} t} \int_{-\infty}^{\infty} u_{1}^{2} \mathrm{~d} \xi=2 R^{2}\left(\delta_{\mathrm{a}}\right) c_{\mathrm{gr}}=4 k_{r}^{2}\left(2 k_{r}^{2}-1\right) R^{2}\left(\delta_{\mathrm{a}}\right) .
$$

When relations (3.18) and (3.19) are substituted into the momentum equation (3.17) and $\epsilon c_{1}$ replaced by $c-c_{\mathrm{ES}}$ (see Eq. (3.4)), we finally obtain the dynamical equation for the perturbed embedded-soliton's velocity $c$ as

$$
\frac{\mathrm{d} c}{\mathrm{~d} t}=-\beta\left(c-c_{\mathrm{ES}}\right)^{2},
$$

where the coefficient $\beta=\Gamma / 2 E$. The solution of Eq. (3.20) is

$$
c(t)=c_{\mathrm{ES}}+\frac{c_{0}-c_{\mathrm{ES}}}{1+\beta\left(c_{0}-c_{\mathrm{ES}}\right) t},
$$

where $c_{0}$ is the initial condition for velocity $c(t)$. The formula for $c_{0}$ can be obtained from Eqs. (3.4) and (3.7) as

$$
c_{0}=c_{\mathrm{ES}}+\frac{\int_{-\infty}^{\infty} U_{\mathrm{ES}}(\xi)\left[u(\xi, 0)-U_{\mathrm{ES}}(\xi)\right] \mathrm{d} \xi}{\int_{-\infty}^{\infty} U_{\mathrm{ES}}(\xi)(\partial U / \partial c)\left(\xi ; c_{\mathrm{ES}}, \delta_{\mathrm{a}}\right) \mathrm{d} \xi} .
$$

Once the initial perturbed embedded soliton $u(\xi, 0)$ is specified, then $c_{0}$ is fixed as above.

The asymptotic equation (3.20) is the key result of this paper. When $\beta>0$, this equation shows that its fixed point $c=c_{\mathrm{ES}}$ is semi-stable: any perturbation with $c_{0}>c_{\mathrm{ES}}$ is stable, and any perturbation with $c_{0}<c_{\mathrm{ES}}$ is unstable. When translated into the original partial differential equation (2.1), it means that the embedded soliton is semi-stable. Depending on the type of initial perturbations, the embedded soliton can persist, or be destroyed.

Finally, when $E$ vanishes, the zero embedded eigenvalue has multiplicity higher than 2, which results in linearized (algebraic) instability of the embedded soliton (see, e.g., [18]). We have excluded such linearized instability in our assumptions above.

\section{Comparison with direct numerical simulations}

In this section, we directly simulate the original partial differential equation (2.1), and compare the results with our analytical theory above. The system parameters we choose are

$$
\alpha_{0}=5, \quad \alpha_{1}=5, \quad \alpha_{2}=2.5, \quad \alpha_{3}=0
$$

in Eq. (2.2). Note that these parameter values are equivalent to $\alpha_{0}=\alpha_{1}=1, \alpha_{2}=0.5$ and $\alpha_{3}=0$ after variable $u$ and time $t$ are rescaled. At these parameter values, Eq. (2.1) is Hamiltonian. The fifth-order long-wave model equation studied by Champneys and Groves [3] corresponds to our equation (2.1) with $\alpha_{0}=1$ and $\alpha_{3}=0$. We have also tested other parameter values with $\alpha_{1}=2 \alpha_{2}$ and found similar results. For instance, in the third-order Hamiltonian long-wave approximation to the water-wave problem as derived by Craig and Groves [26], the parameter values (after variable rescaling) are $\alpha_{0}=1, \alpha_{1}=-5 / 3, \alpha_{2}=-5 / 6$ and $\alpha_{3}=0$. Comparison between our theory and numerics for this set of parameters is qualitatively the same as that for the parameters (4.1).

With the parameters (4.1), the fifth-order KdV equation (2.1) has an embedded soliton

$$
U_{\mathrm{ES}}(x, t)=0.9 \operatorname{sech}^{2}\left[\sqrt{0.3}\left(x-c_{\mathrm{ES}} t\right)\right]
$$

at the exact wave speed $c_{\mathrm{ES}}=2.64$ (see [11]). The approximate phase values $\delta_{\mathrm{s}}$ and $\delta_{\mathrm{a}}$ in the linear modes $\psi_{1,2}$ of the linearization operator $\mathcal{L}$ are found numerically (by the shooting method) as

$$
\delta_{\mathrm{s}}=2.1815, \quad \delta_{\mathrm{a}}=0.5737 .
$$

Note that the difference between these two phases here is not equal to $\pi / 2$, thus the radiation tail amplitude in perturbed embedded solitons is not minimal. However, this phase difference differs from $\pi / 2$ only by 0.037 . Thus 


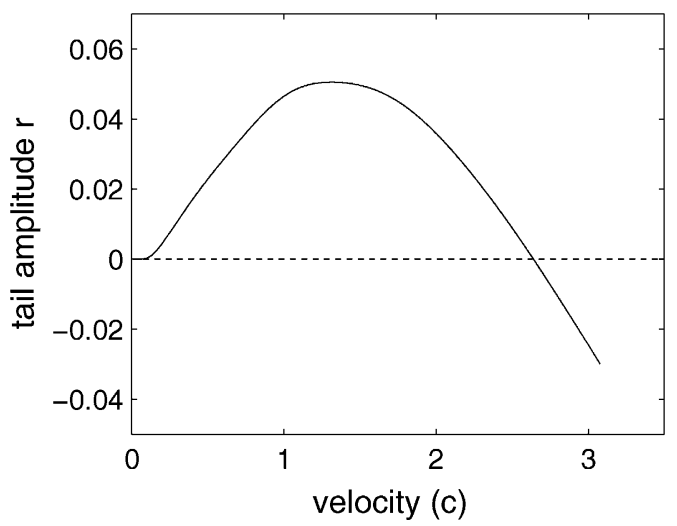

Fig. 1. Tail amplitude $r\left(c, \delta_{\mathrm{a}}\right)$ of symmetric nonlocal waves as a function of velocity $c$. The nonlocal waves satisfy Eq. (2.6) with system parameters (4.1). The radiation phase $\delta=\delta_{\mathrm{a}}$ is the phase of the anti-symmetric linear mode $\psi_{2}$ given by Eq. $(2.15): \delta_{\mathrm{a}} \approx 0.5737$.

the radiation tail amplitude is rather close to its minimal value. At phase $\delta=\delta_{\mathrm{a}}$ (which is the radiation phase), we have numerically obtained the tail amplitude curve $r\left(c, \delta_{\mathrm{a}}\right)$ of symmetric nonlocal waves from Eq. (2.6) at various velocity, $c$, values, again by the shooting method. The results are shown in Fig. 1 . As expected, the tail amplitude $r$ is nonzero for $c>0$ except when $c=c_{\mathrm{ES}}$. The slope $R\left(\delta_{\mathrm{a}}\right)$ at embedded-soliton velocity $c_{\mathrm{ES}}$ is found to be $R\left(\delta_{\mathrm{a}}\right)=-0.0652$. The $k_{r}$ value can be quickly obtained from Eq. (2.7), and the integral in Eq. (3.18) can be readily determined numerically. From all these values, we finally found that $\beta=0.0868$. With this $\beta$ value, our analytical formula for the velocity of perturbed embedded solitons is then given by Eq. (3.21).

In order to verify our analytical theory, we have numerically simulated the original wave equation (2.1) with system parameters (4.1) and initial condition

$$
u(x, 0)=h U_{\mathrm{ES}}(x, 0),
$$

where $h$ is a constant coefficient. Note that $h=1$ gives the exact embedded soliton, and $h \neq 1$ gives a perturbed embedded soliton. Our numerical scheme is the integrating factor method as described in [27]. The $x$ interval is taken as 400 units long, and 1024 grid points are used. The time stepsize is $10^{-4}$. To prevent radiation from re-entering the simulation region through periodic boundary conditions, we have used a damping condition near the boundaries. In our simulation, we have also adopted a frame moving at the embedded-soliton's velocity $c_{\mathrm{ES}}$ (but the results will still be presented in the original frame). Our numerical scheme has been tested with the exact embedded soliton (4.2) as the initial condition. It has also been tested on a related system - the integrable fifth-order KdV hierarchy equation. Furthermore, we have tried different grid points and time stepsizes. These tests show that the numerical error in our scheme is on the order of $10^{-6}$.

We have run our numerical scheme on two typical initial conditions (4.4) with $h=1.05$ and 0.95 . The results are presented in Figs. 2 and 3, respectively. In the former case, the perturbed state has momentum $P$ higher than the embedded soliton's (see Eq. (2.4)). Because of this, the perturbed state initially moves a little faster than the unperturbed embedded soliton (see Fig. 2(b)). But its speed as well as amplitude slowly decrease due to continuous-wave radiation which moves ahead of the main pulse (see Fig. 2(b) and (c)). This tail radiation at $t=20$ is shown in Fig. 2(a). (Note that the tail decay near the right end of the $x$ interval is due to our damping boundary condition. The actual tail length is much longer.) But the tail amplitude decreases also in the process (see Fig. 2(d)). Thus energy radiation is decreasing. Eventually, the perturbed state asymptotically approaches the unperturbed embedded soliton, which is clearly seen in Fig. 2(b) and (c).

When $h=0.95$, the perturbed state has momentum $P$ lower than the embedded soliton's. In this case, due to continuous-wave radiation which intensifies over time (see Fig. 3(a) and (d)), the speed and amplitude of the perturbed embedded soliton both decrease well below their corresponding values of the unperturbed embedded 

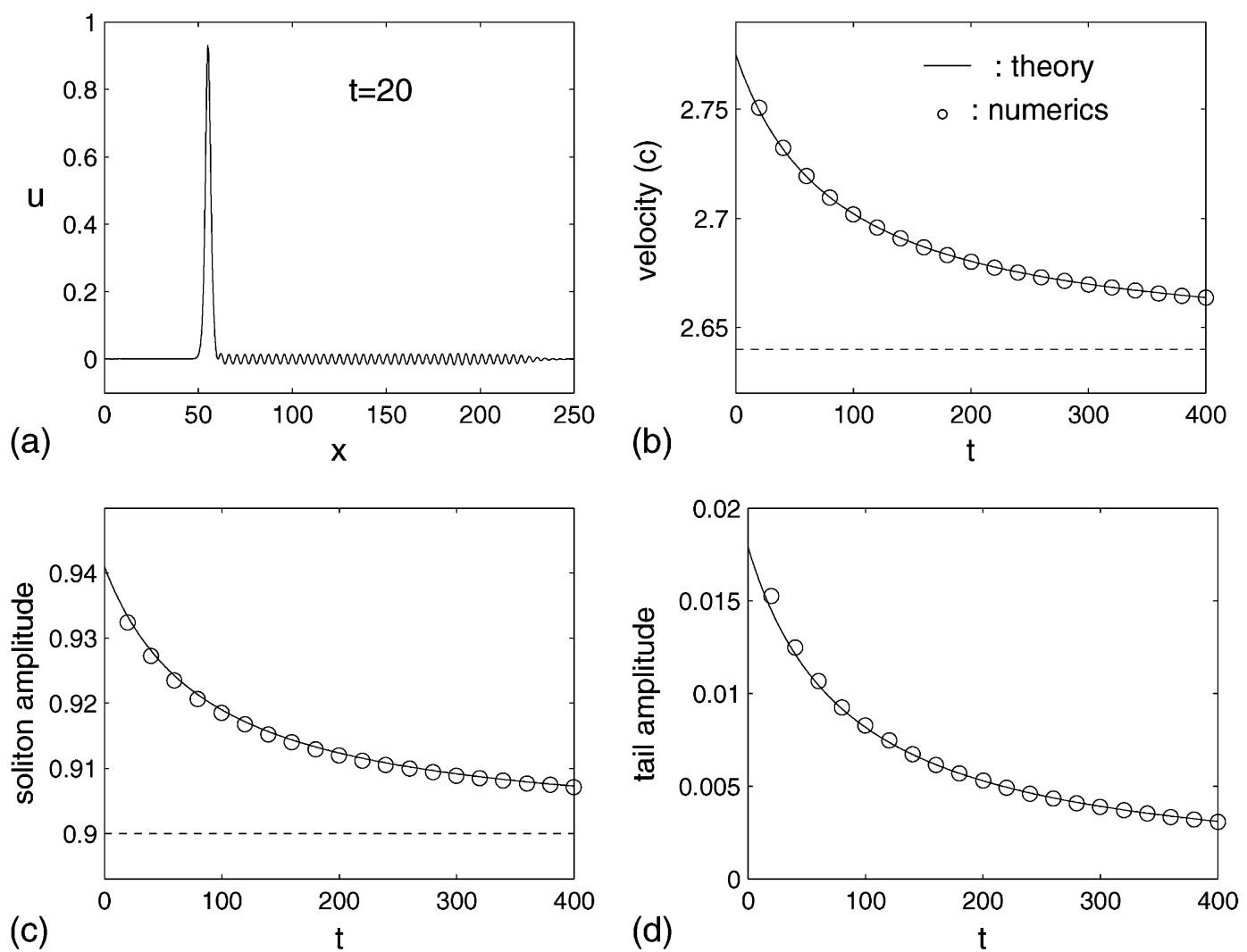

Fig. 2. Evolution of the embedded soliton under the momentum-enhancing perturbation (4.4) with $h=1.05$ : (a) numerical solution profile at $t=20$; (b) velocity $c$ of the perturbed embedded-soliton versus time $t$, the dashed line is the embedded-soliton velocity $c_{\mathrm{ES}}=2.64$; (c) amplitude of the perturbed embedded-soliton versus time $t$, the dashed line is the embedded-soliton amplitude 0.9; (d) radiation tail amplitude versus time $t$. The solid lines in (b)-(d) are theoretical predictions, and circles are numerical values.

soliton (see Fig. 3(b) and (c)). When the amplitude of the main pulse has dropped significantly, it can no longer sustain high radiation tails. Thus tail amplitudes start to decrease (see Fig. 3(d)). Eventually, the embedded soliton is destroyed by perturbations.

The above numerical simulation results agree both qualitatively and quantitatively with our analytical theory. Qualitatively, when $h=1.05$, as the initial velocity is above $c_{\mathrm{ES}}$, formula (3.21) predicts that the pulse velocity will asymptotically approach $c_{\mathrm{ES}}$; when $h=0.95$, the velocity will decay far below $c_{\mathrm{ES}}$. This semi-stability behavior is accurately reflected in the numerical results. Quantitatively, we have also compared the pulse velocity, amplitude and tail amplitude of analytical predictions to those of numerical results. The analytical prediction for pulse velocity is given by formula (3.21). The initial condition $c_{0}$ is calculated from formula (3.22). We found that when $h=1.05$, $c_{0} \approx 2.775$, and when $h=0.95, c_{0} \approx 2.515$. The analytical prediction for the pulse amplitude is the center amplitude of symmetric nonlocal waves at analytically predicted velocity $c$ (see Eq. (3.16)). The analytical prediction for tail amplitude is $\left(c-c_{\mathrm{ES}}\right) R_{\mathrm{rad}}$, where $R_{\mathrm{rad}}$ is given by Eq. (3.14). These analytical predictions have been plotted in Figs. 2(b)-(d) and 3(b)-(d) as well for comparison. In the case $h=1.05$, the quantitative agreement between theory and numerics is excellent at all times. In the other case $h=0.95$, the quantitative agreement is good at the beginning, and gets worse at larger times. The good agreement in the former case is because the main pulse remains close to the embedded soliton at all times, thus the perturbation theory works well. In the latter case, the main pulse deviates significantly from the embedded soliton at large times. When that happens, the perturbation theory breaks down. 

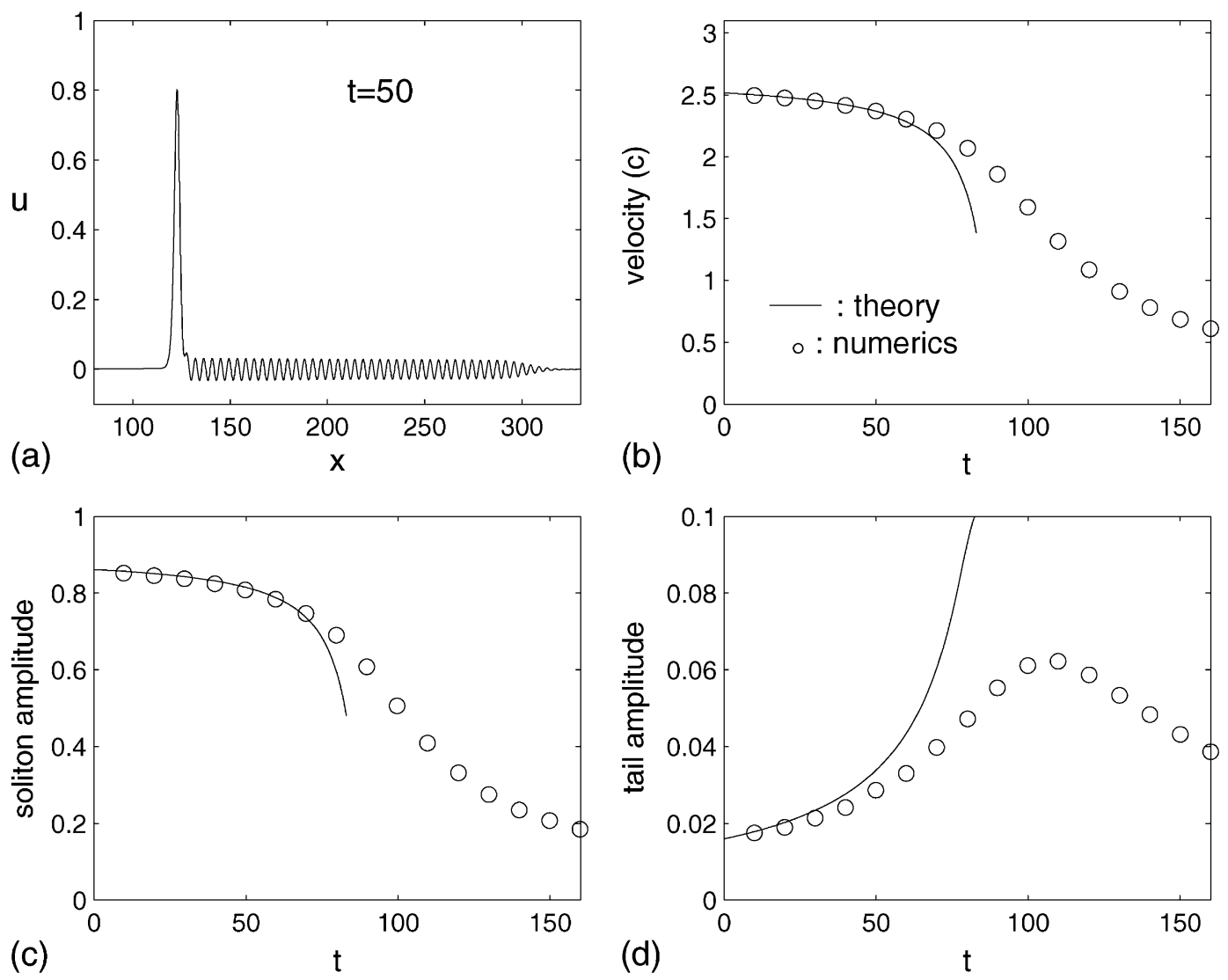

Fig. 3. Evolution of the embedded soliton under the momentum-reducing perturbation (4.4) with $h=0.95$ : (a) numerical solution profile at $t=50$; (b) velocity $c$ of the perturbed embedded-soliton versus time $t$; (c) amplitude of the perturbed embedded-soliton versus time $t$; (d) radiation tail amplitude versus time $t$. The solid lines in (b)-(d) are theoretical predictions, and circles are numerical values.

\section{Conclusion}

In this paper, we have studied the evolution of perturbed embedded solitons in a general Hamiltonian fifth-order $\mathrm{KdV}$ equation (2.1). We have shown that when an embedded soliton is perturbed, it sheds continuous-wave radiation in front of the embedded soliton. The amplitude of this continuous-wave is not minimal in general. Behind the embedded soliton, no flat shelf is created. We have further derived the velocity equation of a perturbed embedded soliton. As a result, the semi-stability property of embedded solitons is analytically proved. In addition, we have obtained the conditions under which a perturbed embedded soliton will decay or persist. We have also simulated the fifth-order $\mathrm{KdV}$ equation numerically. The numerical results agree well with the analysis both qualitatively and quantitatively.

The analysis and the final dynamical equation (3.20) are similar to those found in [14] for generalized SHG models. Thus, in spite of differences in the spectral properties of linearization operators in these two models, the nonlinear resonance between the embedded soliton and the continuous-wave radiation has common features under assumptions listed in Section 3. Obviously, the same method can be applied to any other embedded-soliton-bearing Hamiltonian system. In fact, the system does not even have to be Hamiltonian. A nontrivial conservation law such as power or momentum would be sufficient to guarantee the semi-stability property of embedded solitons (see [14]). 
The open problems going beyond the present study include generation and collisions of several embedded solitons, as well as further engineering applications of embedded solitons in applied science.

\section{Acknowledgements}

The work of YT and JY was supported in part by the Air Force Office of Scientific Research under contract F49620-99-1-0174, and by the National Science Foundation under grant DMS-9971712. The work of DP was supported by NSERC grant 5-36694.

\section{Appendix A}

In this appendix, we present a more rigorous approach for solving the inhomogeneous equation (3.5) for the first-order solution $u_{1}(\xi, t)$ by using a spectral decomposition method for the linearized problem,

$$
(\mathcal{L} \phi(\xi ; k))_{\xi}=\mathrm{i} \Omega(k) \phi(\xi ; k),
$$

where $\Omega(k)=k\left(k^{4}-k^{2}-c_{\mathrm{ES}}\right)$, and $\phi(\xi ; k)$ are continuous-wave eigenfunctions normalized according to the boundary condition

$$
\phi(\xi ; k) \rightarrow \mathrm{e}^{\mathrm{i} k \xi} \quad \text { as } \xi \ll-1 .
$$

The potential terms with $U_{\mathrm{ES}}(\xi)$ decay exponentially at large $|\xi|$ in the operator $\mathcal{L}$ given by Eq. (2.13). The eigenfunctions $\phi(\xi, k)$ may have up to three Fourier oscillatory terms in the limit $\xi \rightarrow+\infty$, which match with the roots of the equation: $\Omega(k)=\Omega$. We will compute asymptotically the Fourier-type integrals (see Eq. (A.10)) at the resonant values $k= \pm k_{r}$, where $k_{r} \equiv k\left(c_{\mathrm{ES}}\right)$ and $k(c)$ is given by Eq. (2.7). At the resonant values, the eigenvalue parameter $\Omega$ is zero, i.e. $\Omega\left( \pm k_{r}\right)=0$. It could be found from Eqs. (2.14) and (2.15) that the boundary condition for $\phi\left(\xi, \pm k_{r}\right)$ in the limit $\xi \rightarrow+\infty$ is

$$
\phi\left(\xi ; \pm k_{r}\right) \rightarrow a_{ \pm} \mathrm{e}^{ \pm \mathrm{i} k_{r} \xi}+b_{ \pm} \mathrm{e}^{\mp \mathrm{i} k_{r} \xi}+c_{ \pm} \quad \text { as } \xi \gg 1,
$$

where

$$
a_{ \pm}=\frac{\mathrm{e}^{ \pm \mathrm{i}\left(\delta_{\mathrm{s}}+\delta_{\mathrm{a}}-\pi / 2\right)}}{\sin \left(\delta_{\mathrm{s}}-\delta_{\mathrm{a}}\right)}, \quad b_{ \pm}=\frac{ \pm \mathrm{i} \cos \left(\delta_{\mathrm{s}}-\delta_{\mathrm{a}}\right)}{\sin \left(\delta_{\mathrm{s}}-\delta_{\mathrm{a}}\right)}, \quad c_{ \pm}=0
$$

Under the assumptions described in Section 3, the solution for $u_{1}(\xi, t)$ can be decomposed through eigenfunctions of the linearized problem (A.1)

$$
u_{1}(\xi, t)=\frac{\partial U}{\partial c}\left(\xi ; c_{\mathrm{ES}}, \delta\right)+\int_{-\infty}^{\infty} w(k, t) \phi(\xi, k) \mathrm{d} k+\alpha U_{\mathrm{ES}}^{\prime}(\xi)
$$

where $\alpha$ is constant. The first term in (A.4) solves the inhomogeneous part of Eq. (3.5). Since the double eigenvalue $\Omega=0$ is embedded into the integral at the resonant points $k= \pm k_{r}$, the inhomogeneous term is not independent and can be decomposed through the same eigenfunctions

$$
\frac{\partial U}{\partial c}\left(\xi ; c_{\mathrm{ES}}, \delta\right)=\int_{-\infty}^{\infty} \frac{F(k) \phi(\xi, k)}{k^{2}-k_{r}^{2}} \mathrm{~d} k,
$$

where $F\left( \pm k_{r}\right) \neq 0$. The singular (pole) part in (A.5) describes the nonlocalized oscillatory tail (2.18) as $|\xi| \gg 1$. With Eq. (A.5) substituted into Eq. (A.4), at time $t=0$, Eq. (A.4) becomes

$$
u_{1}(\xi, 0)=\int_{-\infty}^{\infty} \tilde{w}(k, 0) \phi(\xi, k) \mathrm{d} k+\alpha U_{\mathrm{ES}}^{\prime}(\xi)
$$


where

$$
\tilde{w}(k, 0)=\frac{F(k)}{k^{2}-k_{r}^{2}}+w(k, 0) .
$$

When the initial condition $u_{1}(\xi, 0)$ is localized, then the spectral component $\tilde{w}(k, 0)$ is free of pole singularities.

The complex amplitude $w(k, t)$ in Eq. (A.4) satisfies the trivial evolution equation:

$$
\frac{\partial w}{\partial t}+\mathrm{i} \Omega(k) w=0
$$

Solving the initial-value inhomogeneous problem (3.5) with the spectral decompositions (A.4) and (A.5), we find the following integral representation for $u_{1}(\xi, t)$ :

$$
u_{1}(\xi, t)=\int_{-\infty}^{\infty} \frac{F(k) \phi(\xi, k)}{k^{2}-k_{r}^{2}}\left(1-\mathrm{e}^{-\mathrm{i} \Omega(k) t}\right) \mathrm{d} k+\int_{-\infty}^{\infty} \tilde{w}(k, 0) \mathrm{e}^{-\mathrm{i} \Omega(k) t} \phi(\xi, k) \mathrm{d} k+\alpha U_{\mathrm{ES}}^{\prime}(\xi) .
$$

The second term in Eq. (A.9) represents the nonsingular part produced by the initial condition $u_{1}(\xi, 0)$. The first term in Eq. (A.9) represents the singular (pole) part produced by slow evolution of the embedded soliton. The singular integral term describes the nonlinear resonance between the embedded soliton and the continuous-wave radiation.

The singular (pole) term in Eq. (A.9) occurs for $k= \pm k_{r}$, i.e. when $\Omega\left( \pm k_{r}\right)=0$. This term represents the oscillatory tail radiation that diverges from the embedded soliton with the group velocity $c_{\mathrm{gr}}=\Omega^{\prime}\left(k_{r}\right)=2 k_{r}^{2}\left(2 k_{r}^{2}-\right.$ 1). Since $c_{\mathrm{ES}}>0$ and $k_{r}^{2}>1$, then $c_{\mathrm{gr}}>0$, i.e. the oscillatory tail radiation occurs ahead but not behind of the embedded soliton. We prove this conjecture by using the pole decomposition technique [14]. The singular contribution from the integral (A.9) can be evaluated in the asymptotic region $\xi \ll-1, t \gg 1$ such that $|\xi| / t$ is constant

$$
\begin{aligned}
& \lim _{t \rightarrow+\infty} \int_{-\infty}^{\infty} \frac{F(k) \mathrm{d} k}{k^{2}-k_{r}^{2}} \mathrm{e}^{\mathrm{i} k \xi}\left(1-\mathrm{e}^{-\mathrm{i} k\left(k^{4}-k^{2}-c_{\mathrm{ES}}\right) t}\right) \\
& \quad=\frac{\pi \mathrm{i}}{2 k_{r}}\left(F\left(k_{r}\right) \mathrm{e}^{\mathrm{i} k_{r} \xi}-\bar{F}\left(k_{r}\right) \mathrm{e}^{-\mathrm{i} k_{r} \xi}\right)\left[\operatorname{sign}\left(\frac{\xi}{t}\right)-\operatorname{sign}\left(\frac{\xi}{t}-c_{\mathrm{gr}}\right)\right]=0 .
\end{aligned}
$$

Here, we have used the boundary condition (A.2) and the symmetry relation $\bar{F}\left(k_{r}\right)=F\left(-k_{r}\right)$. Similar but lengthy computations of the integral (A.9) with the boundary condition (A.3) in the region $\xi \gg 1$ prove that the boundary conditions for the solution $u_{1}(\xi, t)$ of Eq. (3.5) in the limit $t \gg+\infty$ and $\xi / t$ held constant is

$$
u_{1}(\xi, t) \rightarrow \begin{cases}R_{\mathrm{rad}} \sin \left(k_{r} \xi+\delta_{\mathrm{rad}}\right) H\left(c_{\mathrm{gr}} t-\xi\right), & \xi \gg 1, \\ 0, & \xi \ll-1,\end{cases}
$$

where $R_{\text {rad }}$ is the radiation amplitude,

$$
R_{\mathrm{rad}}=\frac{2 \pi\left|F\left(k_{r}\right)\right|}{k_{r} \sin \left(\delta_{\mathrm{a}}-\delta_{\mathrm{s}}\right)},
$$

$\delta_{\text {rad }}$ is the radiation phase,

$$
\delta_{\text {rad }}=\arg \left(F\left(k_{r}\right)\right)+\delta_{\mathrm{s}}+\delta_{\mathrm{a}}-\frac{\pi}{2},
$$

and $H(x)$ is the step function, i.e., $H=1$ when $x \geq 0$, and $H=0$ otherwise.

We show that these results are consistent with Eqs. (3.8) and (3.14). Indeed, computing the singular contribution from the integral (A.5) in the region $\xi \ll-1$, we find by similar technique that

$$
\int_{-\infty}^{\infty} \frac{F(k) \mathrm{d} k}{k^{2}-k_{r}^{2}} \mathrm{e}^{\mathrm{i} k \xi}=-\frac{\pi \mathrm{i}}{2 k_{r}}\left(F\left(k_{r}\right) \mathrm{e}^{\mathrm{i} k_{r} \xi}-\bar{F}\left(k_{r}\right) \mathrm{e}^{-\mathrm{i} k_{r} \xi}\right)
$$


Matching this boundary condition with Eq. (2.18) in the region $\xi \ll-1$, we find

$$
\left|F\left(k_{r}\right)\right|=\frac{k_{r}}{\pi} R(\delta), \quad \arg \left(F\left(k_{r}\right)\right)=\pi-\delta .
$$

Let us specify the inhomogeneous solution $\partial U\left(\xi ; c_{\mathrm{ES}}, \delta\right) / \partial c$ at the minimal tail phase $\delta=\delta_{\mathrm{S}}+\pi / 2$, then the homogeneous eigenfunction $\psi_{1}(\xi)$ is excluded from (2.16) and (A.5), since $\gamma_{1}=0$, see below Eq. (2.19). Setting the value $\delta=\delta_{\mathrm{s}}+\pi / 2$ in Eq. (A.15), we finally find from Eqs. (A.12) and (A.13) that

$$
R_{\mathrm{rad}}=2 \frac{R\left(\delta_{\mathrm{s}}+\pi / 2\right)}{\sin \left(\delta_{\mathrm{a}}-\delta_{\mathrm{s}}\right)}=2 R\left(\delta_{\mathrm{a}}\right), \quad \delta_{\mathrm{rad}}=\delta_{\mathrm{a}},
$$

where we have used the relation (2.19).

\section{Appendix B}

In this appendix, we use a local momentum conservation law to derive the dynamical equation for velocity $c$. The local momentum conservation law has the form

$$
\left[\frac{1}{2} u^{2}\right]_{t}+\left[u u_{x x}-\frac{1}{2} u_{x}^{2}+u u_{x x x x}-u_{x} u_{x x x}+\frac{1}{2} u_{x x}^{2}+\frac{2}{3} \alpha_{0} u^{3}+\alpha_{1} u^{2} u_{x x}+\frac{3}{4} \alpha_{3} u^{4}\right]_{x}=0 .
$$

Integrating Eq. (B.1) over $|\xi| \sim \mathrm{O}(1)$ and substituting the perturbation expansion (3.3) up to order $\mathrm{O}\left(\epsilon^{2}\right)$, we derive the following equation:

$$
\begin{aligned}
& \left(\int_{-\infty}^{\infty} U_{\mathrm{ES}} u_{1} \mathrm{~d} \xi\right) \frac{\mathrm{d} c_{1}}{\mathrm{~d} T} \\
& \quad+c_{1}^{2}\left[-\frac{1}{2} c_{\mathrm{ES}} u_{1}^{2}+u_{1} u_{1 \xi \xi}-\frac{1}{2} u_{1 \xi}^{2}+u_{1} u_{1 \xi \xi \xi \xi}-u_{1 \xi} u_{1 \xi \xi \xi}+\frac{1}{2} u_{1 \xi \xi}^{2}\right]_{\xi \rightarrow-\infty}^{\xi \rightarrow+\infty}=0 .
\end{aligned}
$$

The stationary solution $u_{1 \mathrm{~s}}(\xi)$ is given by Eqs. (3.12) and (3.15), and the oscillatory tail in front of the embedded soliton is given by Eqs. (3.8) and (3.14). When those formulas are utilized, we get

$$
\int_{-\infty}^{\infty} U_{\mathrm{ES}} u_{1} \mathrm{~d} \xi=E
$$

and

$$
\left[-\frac{1}{2} c_{\mathrm{ESS}} u_{1}^{2}+u_{1} u_{1 \xi \xi}-\frac{1}{2} u_{1 \xi}^{2}+u_{1} u_{1 \xi \xi \xi \xi}-u_{1 \xi} u_{1 \xi \xi \xi}+\frac{1}{2} u_{1 \xi \xi}^{2}\right]_{\xi \rightarrow-\infty}^{\xi \rightarrow+\infty}=2 k_{r}^{2}\left(2 k_{r}^{2}-1\right) R^{2}\left(\delta_{\mathrm{a}}\right)=\frac{1}{2} \Gamma
$$

where $E$ and $\Gamma$ are defined in Eqs. (3.18) and (3.19). When relations (B.3) and (B.4) are substituted into Eq. (B.2) and $\epsilon c_{1}(T)$ is replaced by $c(t)-c_{\mathrm{ES}}(3.4)$, the dynamical equation (3.20) is reproduced.

\section{References}

[1] S. Kichenassamy, P.J. Olver, Existence and nonexistence of solitary wave solutions to higher-order model evolution equations, SIAM J. Math. Anal. 23 (1992) 1141.

[2] D.C. Calvo, T.R. Akylas, On the formation of bound states by interacting nonlocal solitary waves, Physica D 101 (1997) 270.

[3] A.R. Champneys, M.D. Groves, A global investigation of solitary wave solutions to a two-parameter model for water waves, J. Fluid Mech. 342 (1997) 199.

[4] A.V. Buryak, Stationary soliton bound states existing in resonance with linear waves, Phys. Rev. E 52 (1995) 1156.

[5] J. Fujioka, A. Espinosa, Soliton-like solution of an extended NLS equation existing in resonance with linear dispersive waves, J. Phys. Soc. Jpn. 66 (1997) 2601 
[6] R. Grimshaw, P. Cook, Solitary waves with oscillatory tails, in: A.T. Chwang, J.H.W. Lee, D.Y.C. Leung (Eds.), Proceedings of the Second International Conference on Hydrodynamics, Hong Kong, 1996.

[7] J. Yang, B.A. Malomed, D.J. Kaup, Embedded solitons in second-harmonic-generating systems, Phys. Rev. Lett. 83 (1999) 1958.

[8] A.R. Champneys, B.A. Malomed, M.J. Friedman, Thirring solitons in the presence of dispersion, Phys. Rev. Lett. 80 (1998) 4168.

[9] A.R. Champneys, B.A. Malomed, Moving embedded solitons, J. Phys. A 32 (1999) L547.

[10] A.R. Champneys, B.A. Malomed, Embedded solitons in a three-wave system, Phys. Rev. E 61 (1999) 886.

[11] A.R. Champneys, B.A. Malomed, J. Yang, D.J. Kaup, Embedded solitons: solitary waves in resonance with the linear spectrum, Physica D 152 (2001) 340.

[12] J. Yang, B.A. Malomed, D.J. Kaup, A.R. Champneys, Embedded solitons: a new type of solitary waves, Math. Comput. Simul. 56 (2001) 585.

[13] J. Yang, Dynamics of embedded solitons in the extended KdV equations, Stud. Appl. Math. 106 (2001) 337.

[14] D.E. Pelinovsky, J. Yang, A normal form for nonlinear resonance of embedded solitons, Proc. R. Soc. London A 458 (2002) $1469-1497$.

[15] F. Dias, E.A. Kuznetsov, Nonlinear stability of solitons in the fifth-order Korteweg-de Vries equation, Phys. Lett. A 263 (1999) 98.

[16] S.P. Levandovsky, A stability analysis for firth-order water-wave models, Physica D 125 (1999) 222.

[17] T.J. Bridges, G. Derks, The symplectic Evans matrix, and the instability of solitary waves and fronts, Arch. Rat. Mech. Anal. 156 (2001) 1.

[18] D.E. Pelinovsky, R.H.J. Grimshaw, An asymptotic approach to solitary wave instability and critical collapse in long-wave KdV-type evolution equations, Physica D 98 (1996) 139.

[19] A.R. Champneys, Codimension-one persistence beyond all orders of homoclinic orbits to singular saddle centres in reversible systems, Nonlinearity 14 (2001) 87-112.

[20] R. Grimshaw, B. Malomed, E.S. Benilov, Solitary waves with damped oscillatory tails: an analysis of the fifth-order Korteweg-de Vries equation, Physica D 77 (1994) 473.

[21] E.S. Benilov, R. Grimshaw, E.P. Kuznetsova, The generation of radiating waves in a singularly perturbed KdV equation, Physica D 69 (1993) 270.

[22] R. Grimshaw, N. Joshi, Weakly nonlocal solitary waves in a singularly perturbed Korteweg-de Vries equation, SIAM J. Appl. Math. 55 (1995) 124-135.

[23] V.I. Karpman, E.M. Maslov, Perturbation theory for solitons, Sov. Phys. JETP 46 (1978) 281.

[24] D.J. Kaup, A.C. Newell, Solitons as particles, oscillators, and in slowly changing media: a singular perturbation theory, Proc. R. Soc. London A 361 (1978) 413-446.

[25] J.P. Boyd, Weakly Nonlinear Solitary Waves and Beyond-all-Orders Asymptotics, Kluwer Academic Publishers, Boston, 1998.

[26] W. Craig, M.D. Groves, Hamiltonian long-wave approximations to the water-wave problem, Wave Motion 19 (1994) 367.

[27] P.A. Milewsky, E.G. Tabak, A pseudospectral procedure for the solution of nonlinear wave equations with examples from free-surface flows, SIAM J. Sci. Comp. 21 (1999) 1102. 